\title{
Hábitos alimenticios de poblaciones periféricas de Zaedyus pichiy y Chaetophractus villosus (Cingulata, Chlamyphoridae) en la Patagonia chilena
}

\author{
Aldo Arriagada ${ }^{1,2}$, Luisa Baessolo', Cristián Saucedo³, Julio E. Crespo ,4, Julio Cerda5, Luis Parra², \\ Dennis Aldridge ${ }^{6}$, Jaime Ojeda ${ }^{7}$ \& Alex Hernández ${ }^{8}$
}

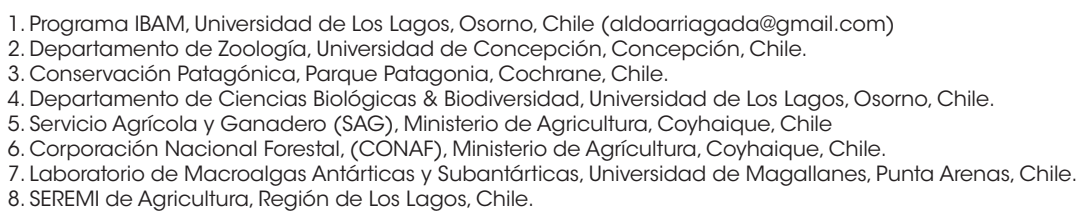

Recibido 31 Marzo 2016

Aceptado 6 Febrero 2017

DOI: 10.1590/1678-4766e2017013

\begin{abstract}
Food habits in peripheral populations of Zaedyus pichiy and Chaetophractus villosus (Cingulata, Chlamyphoridae) in the Chilean Patagonia. In Chile the armadillos Zaedyus pichiy (Desmarest, 1804) and Chaetophractus villosus (Desmarest, 1804) have peripheral populations restricted to steppes and plains near western slopes of the Andes, being also inadequately studied. We present information on feeding habits of both species by analysis of feces obtained in two study areas of Aysén district, Chilean Patagonia. In 38 feces of Z. pichiy we identified two plant taxa, six invertebrate and four vertebrate; in 72 feces of $C$. villosus we identified three plant taxa, nine invertebrate and four vertebrate. In both armadillos species the invertebrates were the category more frequent and diverse. Coleoptera, Orthoptera and Hemiptera were the most abundant insects, and remains of ungulate Lama guanicoe (Müller, 1776) and lagomorph Lepus europaeus (Pallas, 1778) were the most frequent vertebrates. The rarefaction analysis indicates higher taxa richness in $C$. villosus than in $Z$ pichiy and Pianka index indicates there is no significant trophic overlap between these two species of armadillos. Our results indicate omnivores/opportunistic foods habits in peripheral populations of both species, with higher consumption of invertebrates and plants; also they suggest that $C$. villosus lives in a broader environmental range, with a more diverse food habits and higher abundance in both study areas.
\end{abstract}

KEYWORDS. Feces, armadillos, omnivores-scavengers, peripheral distribution.

RESUMEN. En Chile los armadillos Zaedyus pichiy (Desmarest, 1804) y Chaetophractus villosus (Desmarest, 1804) tienen poblaciones periféricas restringidas a planicies de altura y estepa patagónica en la vertiente occidental de la Cordillera de los Andes, donde además han sido escasamente estudiadas. En este trabajo entregamos información sobre los hábitos alimenticios de ambas especies de armadillos, obtenida mediante el análisis de heces colectadas en dos áreas de la región de Aysén, Patagonia chilena. En 38 heces de Z. pichiy identificamos dos taxones de plantas, seis de invertebrados y cuatro de vertebrados, mientras que en 72 heces $C$. villosus identificamos tres, nueve y cuatro taxones de las mismas categorías. En ambas especies de armadillos los invertebrados fueron la categoría más diversa y con mayor frecuencia de ocurrencia, siendo Coleoptera, Orthoptera y Hemiptera los grupos mejor representados en términos de abundancia. Entre los vertebrados se observó mayor frecuencia de restos del camélido Lama guanicoe (Müller, 1776) y el lagomorfo Lepus europaeus (Pallas, 1778). El análisis de rarefacción muestra que la riqueza de taxones fue mayor en C. villosus que en Z. pichiy, y el índice de Pianka indica que no existió un solapamiento trófico significativo entre especies de armadillos. Nuestras observaciones indican que poblaciones periféricas de ambas especies de armadillos muestran dietas omnívoras oportunistas, con un mayor consumo de invertebrados y plantas; además, sugieren que $C$. villosus se desarrolla en un rango ambiental más amplio, con una dieta más diversa y mayor presencia de esta especie en ambas áreas de estudio.

PALABRAS-CLAVE. Heces, armadillos, omnívoros-carroñeros, distribución periférica.

Los armadillos Zaedyus pichiy y Chaetophractus villosus son especies endémicas de Sudamérica (Delsuc et al., 2004), distribuidas simpátricamente en gran parte de sus rangos geográficos y que muestran un solapamiento de sus áreas de distribución de aproximadamente $1.200 .000 \mathrm{~km} 2$ (Fig. 1a). En Chile ambas especies se distribuyen en valles y planicies de altura próximas a la vertiente occidental de la Cordillera de los Andes, además de ambientes esteparios de la Patagonia (IRIARTE, 2007), reconociéndose como el límite de distribución norte para Z. pichiy la región de Valparaíso $\left(32^{\circ} \mathrm{S}\right)$, en tanto que para $C$. villosus este límite corresponde a la región del Biobío $\left(36^{\circ} \mathrm{S}\right)$ (TAMAYo, 2009). Hacia el sur de Chile ambas especies sobreponen parte de sus áreas de distribución hasta el Estrecho de Magallanes $\left(53^{\circ} \mathrm{S}\right)$ (SUPERINa \& ABba, 2014). Aunque en Chile la información poblacional de ambas especies es escasa, es probable que factores antrópicos como la caza ilegal, depredación de perros (domésticos y asilvestrados) y el reemplazo de su 
hábitat natural por suelos de uso ganadero hayan afectado la estabilidad de las poblaciones locales (IRIARTE, 2007).

Los pocos trabajos publicados en Chile indican que ambas especies han sido observadas con mayor frecuencia en los ambientes esteparios de las regiones de Aysén y Magallanes (Gigoux, 1935; MANN, 1978; IRIARTE, 2007). Por otra parte, las dos especies son consideradas históricamente como elementos faunísticos de distribución marginal que han logrado dispersarse gradualmente desde la pampa y estepa patagónica de Argentina (WoLfFSOHN, 1921; MAnN, 1945, 1978; TAMAYo, 2009), modificando sus áreas de distribución en función del hábitat disponible y la expansión-retracción del manto de hielo durante los periodos post-glaciales (PolJAK et al., 2010; AвBA et al., 2014). En la región de Aysén los registros de Z. pichiy y $C$. villosus se concentran en el ecotono formado por la estepa patagónica y el bosque caducifolio de Aysén (LUEBERT \& Pliscoff, 2006), con poblaciones de tamaño indeterminado en algunas áreas silvestres protegidas (Reserva Nacional Lago Carlota, Reserva Nacional Coyhaique y Reserva Nacional Lago Cochrane) (Aldridge \& Alvear, 1987; Mella, 1999; ConAF, 2004). Aunque estas áreas protegidas permiten la conservación de sus poblaciones, la biología de ambas especies de armadillos ha recibido escasa atención en Chile, existiendo reportes aislados de su historia natural (TAMAYO, 2009). Entre las razones que han dificultado el estudio de $Z$. pichiy y $C$. villosus en su ambiente natural, se encuentran sus bajas densidades poblacionales, periodos de actividad crepuscular-nocturno y semi-diurno, conducta tímida frente a la presencia humana y tendencia a vivir en forma solitaria o en grupos reducidos.

Cuando la especie objeto de estudio es difícil de observar en terreno, el análisis de sus hábitos de alimentación utilizando contenidos estomacales y heces, constituyen una alternativa viable para examinar indirectamente las respuestas conductuales, ecológicas y evolutivas de la especie hacia el ambiente (KoHN \& WAYNE, 1997). Estudios que han analizado este tipo de evidencias en $Z$. pichiy y $C$. villosus muestran hábitos omnívoros/carroñeros con dietas diversas compuestas por raíces, frutos, semillas, invertebrados, pequeños vertebrados, entre otros (BRUNO \& CUÉLlAR, 2000; Superina et al., 2009). Considerando la amplitud en la distribución geográfica de ambas especies de armadillos, es probable que las poblaciones chilenas que habitan la periferia occidental de estas distribuciones, manifiesten similitudes o diferencias en el tipo y frecuencia de uso de recursos alimenticios con poblaciones descritas en otras áreas de su distribución, sin embargo, en Chile no existe información sobre sus dietas o patrones de forrajeo.

El objetivo de este trabajo es documentar parte de la historia natural de estas poblaciones chilenas de $Z$. pichiy y $C$. villosus distribuidas en un área geográfica donde su presencia es relativamente común, pero la información publicada es dispersa y escasa. Para ello examinamos y comparamos los hábitos alimenticios de ambas especies de armadillos mediante el análisis de heces colectadas en poblaciones de distribución periférica que habitan en ambientes ecotonales de la Patagonia oriental chilena.

\section{MATERIALES Y MÉTODOS}

Áreas de estudio. El trabajo de campo fue realizado en dos áreas silvestres protegidas del Sur de Chile, incluidas en el proyecto conservacionista Parque Patagonia (http:// www.parquepatagonica.org/), denominadas Estancia Valle Chacabuco (en adelante EVCh; 4707'03'S; 72²9'09'O) y Reserva Nacional Lago Cochrane (en adelante RNLC; 47¹4'30"S; 72³1'25"O). Ambas áreas silvestres comprenden una superficie aproximada de 78.000 ha (Fig. 1b), e incluyen ambientes ecotonales de estepa y bosques fríos de lenga Nothofagus pumilio (Krasser, 1896), además de comunidades arbustivas de menor altura compuestas por ñirre Nothofagus antarctica (Oerst, 1871) y calafate Berberis microphylla (Forst, 1787) (ElBROCH \& WITTMER, 2013a). El clima característico es continental trasandino con degeneración esteparia, gran amplitud térmica con una media anual de $8^{\circ} \mathrm{C}$ y precipitaciones anuales cercanas a $800 \mathrm{~mm}$ (Tome et al., 2007).

Colecta e identificación taxonómica. La dieta fue estudiada mediante heces frescas obtenidas de siete individuos de Z. pichiy y cinco de C. villosus capturados manualmente y de forma oportunista en caminos interiores de las áreas de estudio. Estas capturas fueron autorizadas por el Servicio Agrícola y Ganadero de Chile, Resolución Exenta 6956/2013. Otras 98 heces frescas fueron colectadas en la entrada de madrigueras activas, siendo estas diferenciadas entre las especies de armadillos mediante avistamientos sistemáticos y seguimiento de ejemplares con trampas cámaras. Todos los sitios de colecta (cuatro para $Z$. pichiy y cinco para $C$. villosus) fueron seleccionados considerando información de avistamientos y capturas ocasionales, proporcionada por guardaparques y personal de ambas áreas protegidas. En conjunto, esta información permitió establecer que en las áreas de estudio la población de Z. pichiy está acotada geográficamente al sector Este de EVCh, conocido como Paso Roballos (Fig. 1b), mientras que C. villosus presenta una distribución más amplia, habitando gran parte de EVCh y RNLC. Todas las colectas fueron realizadas entre noviembre de 2012 y junio de 2013 .

En el laboratorio las heces fueron hidratadas, lavadas y cernidas en un tamiz de $2 \mathrm{~mm}$ de apertura de malla, obteniéndose estructuras completas y/o fragmentos de plantas (semillas, fibras de tallos y raíces), invertebrados (antenas, tórax, élitros, pupas) y vertebrados (pelos, plumas, huesos). Dependiendo de la calidad de los restos diagnósticos, estos fueron clasificados en las categorías taxonómicas de especie, género, familia u orden, utilizando colecciones de referencias de flora y fauna colectadas en el área de estudio, además de claves taxonómicas en el caso de los insectos (Toro et al., 2003). Para evaluar el esfuerzo de muestreo calculamos las curvas de acumulación de taxones (COLWELL et al., 2004) para cada especie de armadillo en función del número de heces colectadas, acompañadas de sus respectivas curvas de regresión logística en el programa PAST 2.01 (HAMMER et al., 2001).

Análisis de abundancia de taxones y categorías de alimento. En ambas especies de armadillos todos los 


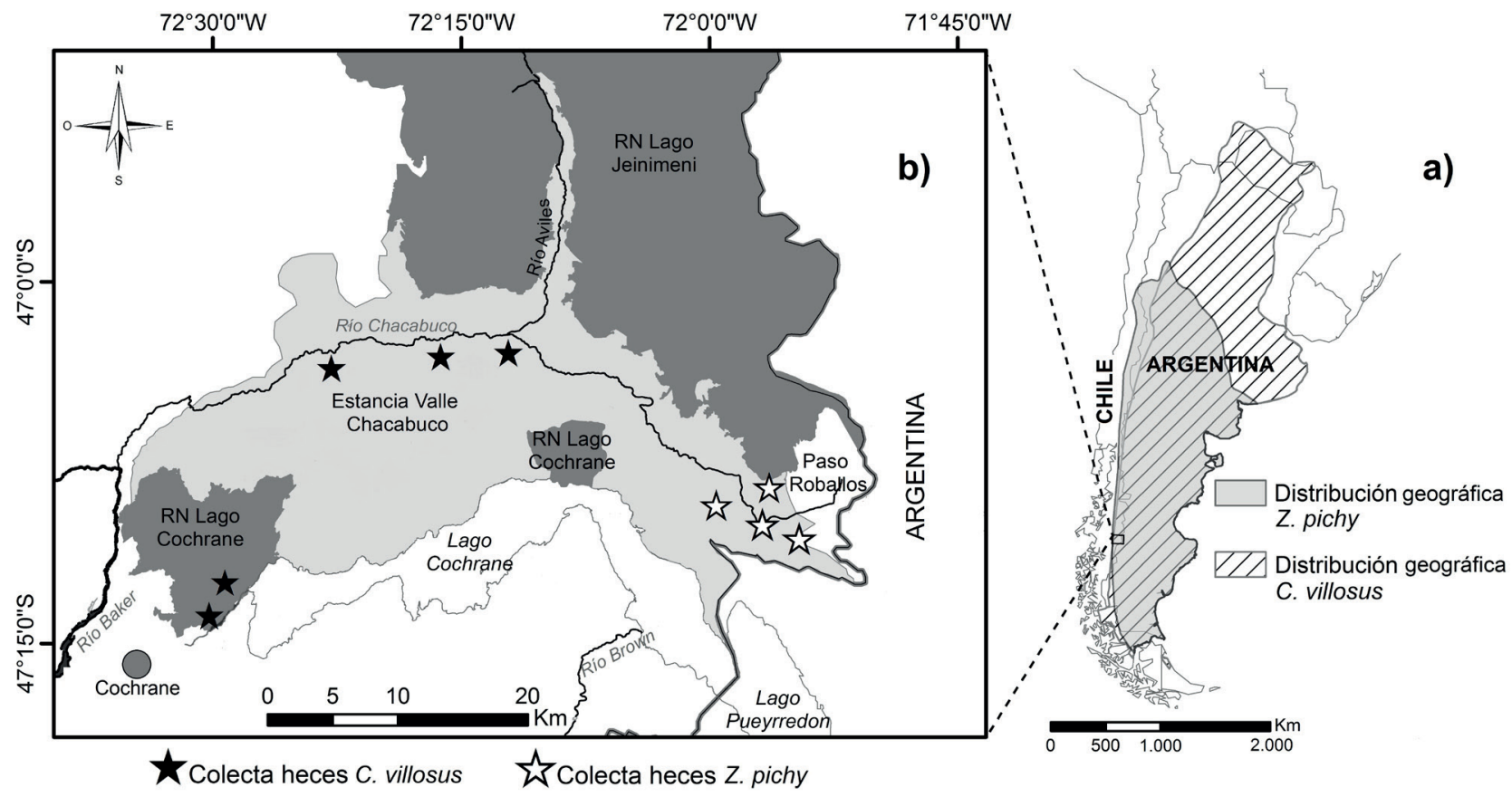

Fig. 1. a) Áreas de distribución geográfica de los armadillos Zaedyus pichiy (Desmarest, 1804) y Chaetophractus villosus (Desmarest, 1804) en Sudamérica; b) ubicación de las áreas de estudio donde se colectaron heces de ambas especies de armadillos.

restos diagnósticos fueron ordenados por mes de colecta para construir una matriz presencia/ausencia de los taxones identificados, obteniéndose además las frecuencias acumuladas de las tres principales categorías de alimento: plantas, invertebrados y vertebrados. Con esta información realizamos un primer análisis para evaluar la incidencia de los distintos taxones de invertebrados y vertebrados (potenciales presas) sobre las dietas de los armadillos, mediante la prueba Kruskall Wallis para medianas (Mdn), implementada en el programa PAST 2.01 (HAMMER et al., 2001). Para esto estimamos valores de abundancia asumiendo que la presencia de un taxón en una hez correspondió a un individuo consumido. En un segundo análisis evaluamos si la frecuencia de ocurrencia de los taxones agrupados en las categorías de plantas, invertebrados y vertebrados se distribuyó homogéneamente sobre el total de muestras obtenidas en cada especie de armadillo o entre meses de colecta, mediante pruebas de bondad de ajuste Chi-cuadrado $\left(\chi^{2}\right)$. Este análisis fue realizado en el programa EcoSim 7.0 (GOTELLI \& ENTSMINGER, 2001), el cual incorpora una prueba de aleatorización que evalúa la hipótesis de independencia entre variables y aleatoriza la matriz de datos, calculando la desviación estándar de Chi-cuadrado de los datos observados y esperados.

Comparación de diversidad y solapamiento trófico. Para contrastar la diversidad de presas entre armadillos se utilizaron curvas de rarefacción, calculándose el mínimo y máximo de taxones esperados de invertebrados y vertebrados bajo los supuestos de un muestreo aleatorio e igualdad de abundancias (HecK et al., 1975). Mediante simulaciones de Monte Carlo se generaron 10.000 pseudomatrices, calculándose la media, varianza e intervalos de confianza del número de taxones. Además, evaluamos el solapamiento dietario entre armadillos con el índice simétrico de Pianka, el cual fluctúa entre 0 (no existen recursos compartidos entre dos especies) y 1 (completa sobreposición de recursos). Para calcular este índice utilizamos la aproximación de modelos nulos, con ausencia de competencia entre especies y el algoritmo de aleatorización RA3 (Niche Breadth Retained/ Zero States Reshuffled; Winemiller \& Pianka, 1990), considerando cada categoría de presa como equiprobable. Se generaron 10.000 pseudomatrices para contrastar estadísticamente el valor observado del índice de Pianka con un promedio esperado. Ambos análisis fueron realizados en el programa EcoSim 7.0 (Gotelli \& ENTSMINGER, 2001).

\section{RESULTADOS}

Identidad taxonómica. En 110 heces colectadas (72 de C. villosus y 38 de Z. pichiy) se obtuvieron 326 restos diagnósticos que permitieron identificar un total de 18 taxones. En las muestras de C. villosus identificamos nueve taxones de invertebrados, cuatro vertebrados y tres plantas; en $Z$. pichiy identificamos seis taxones de invertebrados, cuatro vertebrados y dos plantas (Tab. I). Sobre el total de heces colectadas identificamos seis ordenes de invertebrados, siendo los más diversos Coleoptera con cuatro familias y Orthoptera con dos familias. En mamíferos registramos al ungulado nativo Lama guanicoe (Müller, 1776), especies no determinadas del orden Rodentia y las especies introducidas Lepus europaeus (Pallas, 1778) y Ovis aries (Linnaeus, 1758). Fragmentos de huesos y restos de plumas permitieron la identificación del anseriforme Chloephaga sp. En plantas identificamos a las gramíneas 
nativas Festuca sp. y Pappostipa sp. y el arbusto perenne B. microphylla. Las curvas de acumulaciónon de taxones (Fig. 2) indicaron que las asíntotas fueron obtenidas con 15 heces y 12 taxones en Z. pichiy (representando el 39,5\% del total de heces colectadas) y con 42 heces y 16 taxones en $C$. villosus (58,3\% del total de heces colectadas).

Abundancia de taxones y categorías de alimento. Los taxones de invertebrados que fueron más consumidos por ambas especies de armadillos durante todos los meses de colecta fueron Curculionidae e Hymenoptera (Tab. I). Otros invertebrados consumidos en todos los meses de colecta, pero cuya incidencia fue mayor en muestras de Z pichiy fueron Lucanidae, Carabidae, Diptera y Proscopiidae, y en el caso de C. villosus fueron Lepidotera, Oligochaeta y Acrididae. En vertebrados L. guanicoe y L. europeus mostraron la mayor incidencia en ambas especies de armadillos, pero sus restos no fueron observados durante todos los meses de colecta (Tab. I).

Al comparar la abundancia de taxones de invertebrados y vertebrados en las heces de $Z$. pichiy se observaron diferencias significativas $(\mathrm{n}=83$ individuos, KruskalWallis $=20,76, \mathrm{gl}=9, \mathrm{p}=0,013$ ), donde Curculionidae fue el taxón más abundante con 13 individuos ( $\mathrm{Mdn}=3,5$ individuos, Fig. 3) y los menos abundantes $O$. aries y Rodentia con 3 individuos cada una $(\mathrm{Mdn}=0,5)$. Una tendencia similar fue observada en heces de $C$. villosus $(\mathrm{n}=166$ individuos, KruskalWallis $=30,76, \mathrm{gl}=12, \mathrm{p}=0,002$ ), donde el taxón más abundante fue Curculionidae con 25 individuos $(\mathrm{Mdn}=3,5$, Fig. 3) y el menos abundante Chloephaga sp., con 4 individuos $(\mathrm{Mdn}=0,5)$.
En Z. pichiy las frecuencias de ocurrencia de las categorías de alimento no se distribuyeron homogéneamente $\left(\chi^{2}=1,88, p=0,03\right)$, observándose un mayor registro de invertebrados (69 observaciones), seguido de plantas (26) y vertebrados (14). Además, la sumatoria de todas las categorías difirió significativamente entre meses de colecta $\left(\chi^{2}=1,44\right.$, $\mathrm{p}=0,0001$ ), acumulándose el máximo de registros en marzo de 2013 (32) y el mínimo en noviembre de 2012 (16). En $C$. villosus se observó similar tendencia $\left(\chi^{2}=4,07, \mathrm{p}=0,0001\right)$, con mayor presencia de invertebrados (136), seguido de plantas (51) y finalmente vertebrados (30), existiendo además diferencias significativas entre meses $\left(\chi^{2}=4,08, \mathrm{p}=0,005\right)$, acumulándose el máximo en enero de 2013 (50) y el mínimo en noviembre de 2012 (18).

Diversidad y solapamiento trófico. En heces de $C$. villosus identificamos un total de 16 taxones y 217 individuos, y en Z. pichiy identificamos 12 taxones y 109 individuos. De acuerdo al análisis de rarefacción esta diferencia de diversidad es significativa, dado que el mínimo y máximo teórico de taxones que C. villosus alcanzó con 109 individuos fue de 15 y 16 respectivamente, resultando en ambos casos mayor al valor máximo observado en $Z$. pichiy con igual abundancia (Fig. 4). Este resultado es concordante con el índice de Pianka, para el cual se obtuvo un valor observado de 0,67 y 1.235 pseudomatrices simuladas menores o iguales a este valor (Fig. 5); posteriormente, al comparar el valor observado con el promedio esperado por azar $(0,59)$ no se observaron diferencias significativas $(\mathrm{p}=0,17)$, indicando que no existió solapamiento de presas entre Z. pichiy y C. villosus.

Tab. I. Ocurrencia de ítems alimenticios registrados en las heces de Zaedyus pichiy (Desmarest, 1804) y Chaetophractus villosus (Desmarest, 1804) colectadas en EVCh y RNLC, Patagonia chilena.

\begin{tabular}{|c|c|c|c|c|c|c|c|c|c|c|}
\hline \multirow{2}{*}{ Taxones } & \multicolumn{4}{|c|}{ Zaedyus pichiy } & \multicolumn{6}{|c|}{ Chaetophractus villosus } \\
\hline & Nov & Ene & Feb & Mar & Nov & Dic & Ene & Feb & Mar & Jun \\
\hline \multicolumn{11}{|l|}{ Poaceae } \\
\hline Festuca sp. & 0 & 0 & 0 & 0 & 1 & 1 & 0 & 3 & 4 & 4 \\
\hline Pappostipa sp. & 3 & 1 & 3 & 3 & 2 & 2 & 5 & 4 & 0 & 2 \\
\hline \multicolumn{11}{|l|}{ Berberidaceae } \\
\hline Berberis microphylla & 3 & 4 & 4 & 2 & 0 & 3 & 4 & 6 & 0 & 0 \\
\hline Plantas no identificadas & 0 & 2 & 0 & 1 & 2 & 2 & 0 & 0 & 2 & 4 \\
\hline \multicolumn{11}{|l|}{ Coleoptera } \\
\hline Carabidae & 1 & 2 & 4 & 3 & 1 & 0 & 3 & 2 & 0 & 2 \\
\hline Curculionidae & 2 & 3 & 4 & 4 & 3 & 3 & 6 & 4 & 2 & 7 \\
\hline Lucanidae & 2 & 3 & 3 & 4 & 0 & 5 & 4 & 2 & 1 & 4 \\
\hline Scarabaeidae & 0 & 0 & 0 & 0 & 0 & 2 & 2 & 2 & 1 & 3 \\
\hline Diptera & 1 & 3 & 3 & 5 & 0 & 0 & 0 & 0 & 0 & 0 \\
\hline Hymenoptera & 2 & 3 & 3 & 4 & 3 & 2 & 5 & 6 & 2 & 2 \\
\hline Lepidoptera & 0 & 0 & 0 & 0 & 2 & 3 & 4 & 4 & 2 & 5 \\
\hline Oligochaeta & 0 & 0 & 0 & 0 & 1 & 3 & 3 & 3 & 0 & 2 \\
\hline \multicolumn{11}{|l|}{ Orthoptera } \\
\hline Acridae & 0 & 0 & 0 & 0 & 2 & 1 & 6 & 3 & 2 & 5 \\
\hline Proscopiidae & 1 & 4 & 2 & 3 & 0 & 2 & 2 & 0 & 0 & 2 \\
\hline \multicolumn{11}{|l|}{ Vertebrata } \\
\hline Rodentia & 0 & 1 & 0 & 2 & 1 & 1 & 1 & 0 & 1 & 2 \\
\hline Lepus europaeus & 1 & 3 & 1 & 0 & 0 & 1 & 2 & 3 & 0 & 2 \\
\hline Lama guanicoe & 0 & 1 & 2 & 1 & 0 & 2 & 3 & 2 & 1 & 4 \\
\hline Ovis aries & 0 & 0 & 2 & 0 & 0 & 0 & 0 & 0 & 0 & 0 \\
\hline Chloephaga sp. & 0 & 0 & 0 & 0 & 0 & 0 & 0 & 2 & 1 & 1 \\
\hline $\mathrm{N}^{\circ}$ de heces/mes & 8 & 6 & 11 & 13 & 12 & 10 & 13 & 12 & 7 & 18 \\
\hline
\end{tabular}




\section{DISCUSION}

Heces de ambas especies de armadillos colectadas en EVCh y RNLC presentaron mayor ocurrencia de invertebrados, seguido de plantas y vertebrados, una tendencia reiterada durante todo el periodo de estudio. De acuerdo a otras investigaciones el mayor consumo de invertebrados es un hábito alimenticio general compartido con otros cingulados, como: Cabassous unicinctus (Linnaeus, 1758), Chaetophractus vellerosus (Gray, 1865), Dasypus hybridus (Desmarest, 1804), Dasypus novemcinctus (Linnaeus, 1758), Euphractus sexcinctus (Linnaeus, 1758), Priodontes maximus (Kerr, 1792) y Tolypeutes matacus (Desmarest, 1804) (Bolković et al., 1995; TyLER et al., 1996; ANACLETO \& Marinho-Filho, 2001; Dalponte \& Tavares-Filho, 2004; Anacleto, 2007; Soibelzon et al., 2007; АвBA et al., 2011a,b; WhITAKER et al., 2012). Con respecto a otras poblaciones de Z. pichiy, nuestros resultados concuerdan con los reportados en una población de distribución central localizada en la provincia de Mendoza, Argentina (35 40'S; $68^{\circ} 36^{\circ} \mathrm{O}$ ), donde el análisis de contenidos estomacales indicó mayor incidencia de invertebrados, identificando a Coleoptera como el grupo predominante y más diverso (SUPERINA et al., 2009). No obstante, en el trabajo de Superina et al. (2009) los coleópteros más abundantes correspondieron a las familias Scarabaeidae y Tenebrionidae, mientras que nuestros resultados indican que en ambas especies de armadillos predominaron las familias de coleópteros Curculionidae y Lucanidae (Tab. I; Fig. 3). Otro estudio realizado en poblaciones argentinas de Z. pichiy y C. villosus que habitan ambientes pampeanos próximos a Bahía Blanca $\left(38^{\circ} 35^{\prime} \mathrm{S} ; 62^{\circ} 19^{\prime} \mathrm{O}\right)$, indican que la materia vegetal fue la categoría más importante en sus heces, seguido de coleópteros e himenópteros (M. Ciuccio, datos no publicados). Resultados similares se reportaron para una población periférica de $C$. villosus en el Chaco Boliviano (1950'S; 62 $40^{\circ} \mathrm{O}$ ), donde el análisis de contenidos estomacales determino que los restos vegetales representaron el 59\% de la dieta (identificándose siete especies de plantas, incluidas leguminosas, cucurbitáceas y cactáceas), mientras que los invertebrados representaron el $38 \%$ de la misma (BRUNo \& Cú́llar, 2000). Por nuestra parte, en ambas especies de armadillos sólo identificamos tres especies de plantas, pero es probable que su diversidad se encuentre subestimada debido al alto grado de degradación de tallos, hojas y frutos en las heces analizadas. En este mismo acápite, es interesante destacar el potencial rol de ambas especies de armadillos como agentes dispersores de plantas como B. microphylla, un arbusto espinoso nativo de amplia distribución en la Patagonia, que produce una baya comestible y cuyas semillas fueron registradas en las heces colectadas durante el periodo estival. Observaciones adicionales sugieren una asociación entre la presencia de insectos (adultos y estados inmaduros) y las heces colectadas en madrigueras próximas a sitios con abundante vegetación nativa como Festuca pallescens (St. Ives, 1952), Mulinum spinosum (Pers, 1805) y Baccharis patagonica (Hook \& Arn, 1841), bosques de fagáceas con arbustos de B. microphylla y

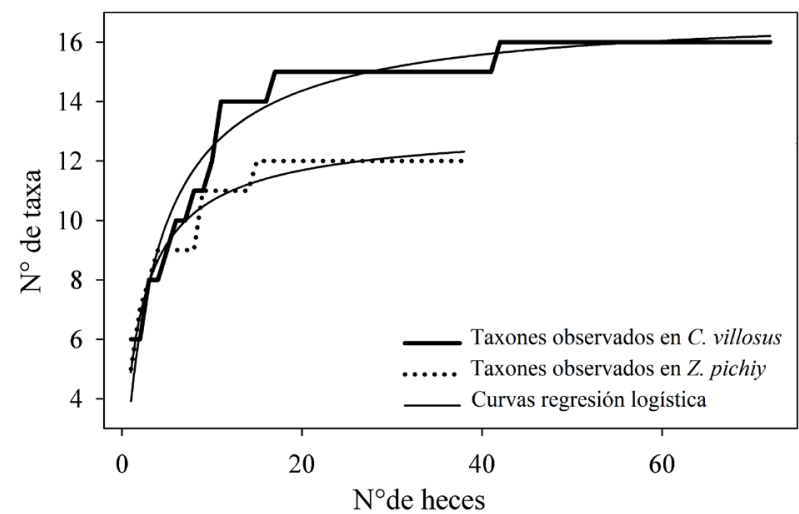

Fig. 2. Curvas de acumulación de taxones identificados en heces de Zaedyus pichiy (Desmarest, 1804) $(\mathrm{n}=38)$ y Chaetophractus villosus (Desmarest, 1804) $(\mathrm{n}=72)$ en EVCh y RNLC, Patagonia chilena. Las líneas continuas representan las regresiones logísticas obtenidas a partir de los datos observados.

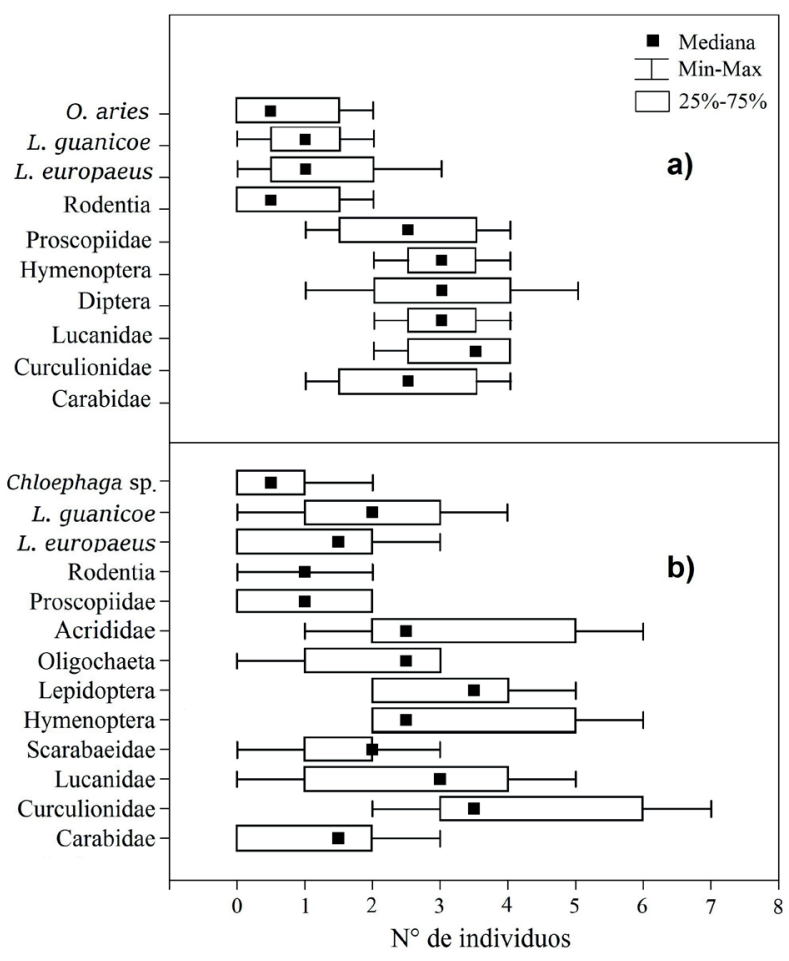

Fig. 3. Gráficos boxplot que representan la abundancia de taxones identificados en heces de a) Zaedyus pichiy (Desmarest, 1804) y b) Chaetophractus villosus (Desmarest, 1804) en EVCh y RNLC, Patagonia chilena.

riberas de esteros, donde observamos altas concentraciones de hozaduras.

El registro de mamíferos y aves en las heces puede relacionarse con el consumo de cadáveres en estados tempranos de descomposición o una ingesta incidental derivada de la búsqueda y consumo de la entomofauna necrófaga que coloniza los cadáveres (TALMAGE \& BUCHANAN, 1954; SUPERINA et al., 2009). En sectores intermedios de EVCh observamos con regularidad carcasas de L. guanicoe, especie de camélido cuyas poblaciones han mostrado una 
recuperación significativa durante los últimos años, producto de la restauración ecológica de esta extensa área geográfica que durante gran parte del siglo XX funcionó como estancia ganadera (ElbRoch \& WitTMER, 2013a). Investigaciones realizadas en esta área de estudio indican que L. guanicoe es una de las presas consumidas con mayor frecuencia por la población local de pumas Puma concolor (Linnaeus, 1771), siendo sus carcasas aprovechadas en forma secundaria por zorros y aves rapaces (ELBROCH \& WITTMER, 2012, 2013a,b). Concordante con lo anterior, mediante observaciones complementarias registramos dos individuos de C. villosus alimentándose intermitentemente de cadáveres de L. guanicoe durante el crepúsculo y amanecer, realizando hozaduras de poca profundidad y defecando en las proximidades de las carcasas. Otro resto de vertebrado común en heces de ambos armadillos correspondió al lagomorfo L. europaeus, especie introducida en Sudamérica a fines del siglo XIX (BonINo et al., 2010) y considerada en Chile como una presa frecuente de pumas, zorros, aves rapaces nocturnas y diurnas (FrANKLIN et al., 1999; YAÑEZ et al., 2009). El efecto de $L$. europaeus en especies de vertebrados nativos fue evaluado en la Patagonia Argentina por TRAVAINI et al. (1998), quienes estudiaron la dinámica en el consumo de un ensamble de carroñeros sobre las carcasas de este lagomorfo. Estos autores observaron que carcasas de L. europaeus son consumidas principalmente por aves rapaces en un periodo de uno a dos días, desplegando conductas gregarias y agresivas para obtener el recurso, e interfiriendo activamente el consumo de otros vertebrados como C. villosus y Z. pichiy, especies que se alimentaban en ausencia de rapaces y por periodos breves tiempo (TRAVAINi et al., 1998). Las observaciones de estos autores complementan las nuestras, sugiriendo que el consumo de carroña vertebrada por parte de estas especies de armadillos es de carácter limitado y ocasional, situación que puede estar relacionada con factores como el tamaño corporal de los cadáveres, frecuencia de ocurrencia de carcasas en un área determinada y diversidad del ensamble local de carroñeros (e.g., SElva et al., 2005; Moleón et al., 2015).

El registro de los taxones Curculionidae e Hymenoptera en las heces de ambas especies de armadillos durante todos los meses de colecta, sugiere que son insectos conspicuos en los ambientes donde forrajean las poblaciones estudiadas, constituyendo además ítems alimenticios recurrentes en sus dietas (Tab. I). Estos grupos y otros como Lucanidae, Carabidae, Lepidoptera, Acrididae o Proscopiidae se encuentran ampliamente distribuidos en variados ambientes del sur de Chile, con estados larvales, juveniles y adultos presentes horizontes superficiales del suelo y parches de vegetación donde obtienen alimento (PEÑA, 1988; RoJAs \& GALlARDO, 2004). En cuanto a los taxones de vertebrados, concluimos que ambas especies de armadillos consumieron con mayor frecuencia en las áreas de estudio restos de $L$. guanicoe, L. europaeus y ocasionalmente roedores (Tab. I). Restos del ovino $O$. aries fueron registrados en un pequeño número de heces colectadas en el Paso Fronterizo Roballos, donde convergen predios ganaderos y se observan regularmente cadáveres de ovinos atribuibles a la depredación de pumas y zorros.

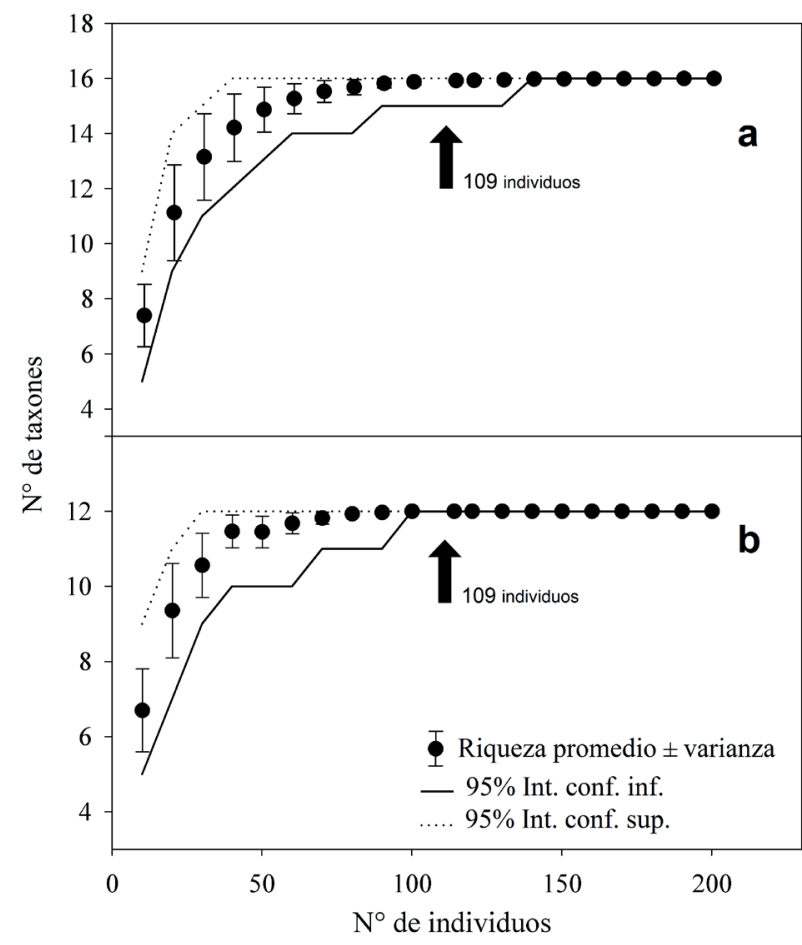

Fig. 4. Curvas de rarefacción de taxones, calculadas para a) Chaetophractus villosus (Desmarest, 1804) y b) Zaedyus pichiy (Desmarest, 1804) en EVCh y RNLC, Patagonia chilena. Se observa que C. villosus ponderó con 109 individuos una riqueza más alta que la observado en $Z$. pichiy con igual abundancia.

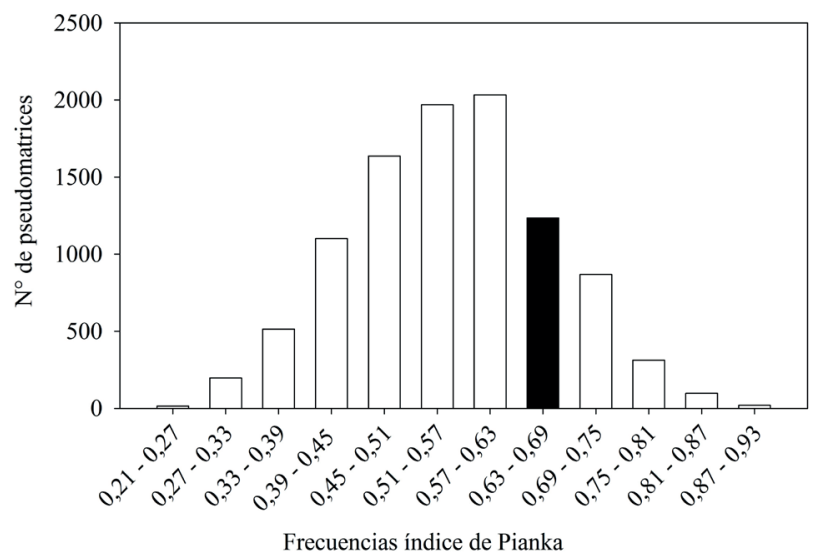

Fig. 5. Distribución de frecuencia del Índice de Pianka obtenida con simulaciones de Monte Carlo. La barra negra indica el intervalo donde se registro el valor observado del índice $(0,67)$.

La comparación de ítems presa mediante el análisis de rarefacción indica que la diversidad de invertebrados y vertebrados fue significativamente mayor en $C$. villosus que en $Z$. pichiy (16 versus 12 taxones; Fig. 4). Aunque compartieron el $50 \%$ de taxones de invertebrados y el $60 \%$ de taxones de vertebrados, el índice de Pianka indica que no existió un solapamiento significativo de ítems alimenticios entre estas especies de armadillos (Fig. 5). En términos biológicos estos resultados se relacionan con la diferencia en la diversidad de recursos alimenticios disponibles en 
los ambientes ecotonales donde se colectaron las heces (KITZBERGER, 2012), y además, proporcionan una estimación cualitativa sobre como las especies estudiadas utilizan estos recursos. Cabe destacar que estos armadillos muestran similitud en sus estrategias de alimentación, pero exhiben diferencias en el periodo de actividad diaria realizada en el exterior de sus madrigueras, siendo Z. pichiy descrita como una especie de hábitos diurnos (SUPERINA et al., 2009) y $C$. villosus como de hábitos crepusculares y nocturnos (TAMAYO, 2009); no obstante, también se ha planteado que el rango de actividad en $C$. villosus varía en función de la localidad geográfica y la estacionalidad (IRIARTE, 2007; ABBA \& CASSINI, 2008). Por otro lado, en términos estadísticos los resultados podrían estar afectados por la comparación de muestras de distinto tamaño ( 72 heces versus 38 heces), y aunque intentamos colectar más heces de Z. pichiy para homologar el tamaño muestral de C. villosus, no fue posible debido a que la última especie mostró mayor abundancia en ambos sitios de estudio, redundando en un mayor número de muestras. No obstante, para ambas especies, las curvas de acumulación de presas alcanzaron nivel asintótico analizando menos del 60\% de sus muestras (Fig. 2), sugiriendo que el esfuerzo de muestreo fue adecuado para describir y comparar los ítems alimenticios.

Nuestras observaciones de campo y el análisis de las heces sugieren que las poblaciones periféricas de $C$. villosus se desarrollan en un rango ambiental más amplio que sus símiles de Z. pichiy, evidenciado a través de una dieta más diversa y la presencia de individuos y madrigueras activas en ambas áreas estudiadas. Como fue mencionado en la metodología, las poblaciones de $C$. villosus se distribuyen en gran parte del valle de ecotonal de la cuenca del río Chacabuco, desde Paso Roballos hasta los ambientes suburbanos de la ciudad de Cochrane ( $\left.47^{\circ} 15^{\prime} \mathrm{S} ; 72^{\circ} 34^{\prime} \mathrm{O}\right)$, incluidos algunos avistamientos en la ribera Oeste del caudaloso río Baker $\left(47^{\circ} 16^{\prime} \mathrm{S} ; 7^{\circ} 44^{\prime} \mathrm{O}\right)$ (Aldo Arriagada, datos no publicados). En dirección Este a Oeste, la transición ambiental de este valle ecotonal incluye condiciones semiáridas de estepa herbácea con coironales y matorral bajo, humedales, bosque caducifolio de fagáceas y bosque siempre verde mixto presente en la cuenca superior del río Baker (Luebert \& Pliscoff, 2006; Elbroch \& WittMer, 2012). Por otro lado, la población de Z. pichiy se encuentra acotada a la estepa adyacente a las planicies patagónicas argentinas en el sector Este de EVCh, y durante la realización del trabajo de campo no se registro evidencia de dispersión de individuos hacia el sector Este del mencionado valle. De acuerdo a lo anterior, nuestros resultados describen parte de la plasticidad ecológica que muestran dos especies de armadillos que habitan en condiciones ambientales limites dentro de sus áreas de distribución, donde teóricamente los recursos disponibles son de menor calidad con respecto a los existentes en las áreas de distribución central (HoffmanN \& Blows, 1994; Zielinski \& DunCAN, 2004). En especies con extensas distribuciones geográficas como las estudiadas, los factores ambientales varían a menudo en forma clinal, encontrándose las poblaciones periféricas propensas a experimentar diferentes escenarios de selección natural (ENDler, 1977; Lesica \& AlLendorf, 1995), y pueden ser relevantes en la conservación de las especies debido a su diversidad genética y rol fundamental en la especiación, supervivencia y expansión de las especies durante los cambios ambientales drásticos (Fraser, 2000; Hampe \& Petit, 2005).

En síntesis, este trabajo constituye el primer reporte sobre la dieta de Z. pichiy y C. villosus para la Patagonia chilena, mostrando que al igual que otras especies de armadillos consumen un amplio espectro de alimentos, con preferencia por el consumo de invertebrados. Finalmente sugerimos que a nivel local es necesario investigar otros ámbitos de la historia natural de estas especies, considerando que forman parte del grupo inicial de mamíferos placentarios que radiaron en Sudamérica en el límite del Cretácico TardíoTemprano (Delsuc et al., 2004; GiBB et al., 2015), y que en Chile presentan una distribución marginal, por lo que constituyen modelos biológicos adecuados para evaluar los mecanismos de adaptación y expansión de los organismos en la Patagonia.

Agradecimientos. Los autores agradecen a Conservación Patagónica, CONAF y SAG por las autorizaciones y apoyo logístico para trabajar en los sitios de estudio. Además, extendemos nuestros agradecimientos a los revisores anónimos por sus sugerencias, comentarios y críticas constructivas hacia el manuscrito. Aldo Arriagada agradece el apoyo de CONICYT y la Dirección de Postgrado de la Universidad de Concepción. Este trabajo es parte del proyecto Los armadillos y su presencia en la estepa aisenina: descubriendo la historia natural de un grupo endémico de mamíferos sudamericanos financiado por el Fondo de Protección Ambiental, Ministerio del Medio Ambiente-Chile.

\section{REFERENCIAS BIBLIOGRÁFICAS}

ABBA, A. \& CASsini, M. 2008. Ecology and conservation of three species of armadillos in the Pampas region, Argentina. In: Loughry, W. \& Vizcaíno, S. eds. The Biology of the Xenarthra. Gainesville, University Press of Florida, p. 300-305.

AbBa, A.; Cassini, G. \& Galliari, F. 2011a. Nuevos aportes a la historia natural de la mulita pampeana Dasypus hybridus (Mammalia: Dasypodidae). Iheringia, Série Zoologia 101(4):325-335.

AbBa, A.; Cassini, G.; CAssini, M. \& Vizcaíno, S. 2011b. Historia natural del piche llorón Chaetophractus vellerosus (Mammalia: Xenarthra, Dasypodidae). Revista Chilena de Historia Natural 84(1):51-64.

Abba, A.; PoljaK, S.; Gabrielli, M.; Teta, P. \& Pardiñas, U. 2014. Armored invaders in patagonia: recent southward dispersion of armadillos (Cingulata, Dasypodidae). Mastozoología Neotropical 21(2):311-318.

Aldridge, D. \& AlveAr, N. 1987. Resumen de antecedentes de flora y fauna en la XI región de Aysén. CONAF, Chile. Publicación de Divulgación 13(11):1-67.

AnACleto, T. 2007. Food habits of four armadillo species in the Cerrado Area, Mato Grosso, Brazil. Zoological Studies 46(4):529-537.

AnACleto, T. \& MARINHO-Filho, J. 2001. Hábito alimentar do tatu-canastra (Xenarthra, Dasypodidae) em uma área de cerrado do Brasil Central. Revista Brasileira de Zoologia 18(3):681-688.

Bolković, M.; Caziani, S. \& Protomastro, J. 1995. Food habits of Three Banded Armadillo (Xenarthra: Dasypodidae) in the dry Chaco, Argentina. Journal of Mammalogy 76(4):1199-1204.

Bonino, N.; Cossíos, D. \& Menegheti, J. 2010. Dispersal of the european hare, Lepus europaeus in South America. Folia Zoologica 59(1):9-15.

BRUno, N. \& CuÉllar, E. 2000. Hábitos alimenticios de cinco armadillos en el Chaco Boliviano. In: Cabrera, E.; Mercolli, C. \& Resquin, R. eds. Manejo de fauna silvestre en Amazonía y Latinoamérica. Asunción, Fundación Moisés Bertoni, University of Florida, p. 401-411. 
Colwell, R.; MaO, C. \& Chang, J. 2004. Interpolating, extrapolating, and comparing incidence-based species accumulation curves. Ecology 85(10):2717-2727.

ConAf. 2004. Plan de Manejo Reserva Nacional Lago Cochrane y lotes aledaños, región de Aysén, Chile. Documento de Trabajo 406:1-155.

Dalponte, J. \& TAVAres-Filho, J. 2004. Diet of the yellow armadillo Euphractus sexcinctus in South-Central Brazil. Edentata 6:37-41.

Delsuc, F.; Vizcaíno, S. \& Douzery, E. 2004. Influence of Tertiary paleoenvironmental changes on the diversification of South American mammals: a relaxed molecular clock study within xenarthrans. BMC Evolutionary Biology 4:11.

Elbroch, L. \& Wittmer, H. 2012. Puma spatial ecology in open habitats with aggregate prey. Mammalian Biology 77(5):377-384.

Elbroch, L. \& WitTmer, H. 2013a. The effects of puma prey selection and specialization on less abundant prey in Patagonia. Journal of Mammalogy 94(2):259-268.

Elbroch, L. \& Wittmer, H. 2013b. Nuisance ecology: do scavenging condors exact foraging costs on pumas in Patagonia? PLoS ONE 8:e53595 doi:10.1371/journal.pone.0053595.

ENDLER, J. 1977. Geographic variation, speciation and clines. Princeton, Princeton University Press. 246p.

Franklin, W.; Johnson, W.; Sarno, R. \& Iriarte, A. 1999. Ecology of the Patagonia puma Felis concolor patagonica in southern Chile. Biological Conservation 90:33-40.

Fraser, D. 2000. Species at the edge: the case for listing of peripheral species. In: DARling, L. ed. Proceedings of a Conference on the Biology and Management of Species and Habitats at Risk. Victoria and Kamloops, Ministry of Environment, Lands and Parks, and University College of the Cariboo, p. 49-53.

Gibb, G.; Condamine, F.; Kuch, M.; EnK, J.; Moraes-Barros, N.; Superina, M.; Poinar, H. \& Delsuc, F. 2015. Shotgun mitogenomics provides a reference phylogenetic framework and timescale for living Xenarthrans. Molecular Biology and Evolution 33(3):621-642

Gigoux, E. 1935. Zoología de Aysén. Boletín Museo Nacional de Historia Natural, Chile 15:59-63.

Gotelli, N. \& Entsminger, G. 2001. EcoSim: Null models software for ecology. Version 7.0. Acquired Intelligence Inc. \& Kesey-Bear. http:// homepages.together.net/ gentsmin/ecosim.htm.

Hampe, A. \& Petit, R. 2005. Conserving biodiversity under climate change: the rear edge matters. Ecology Letters 8(5):461-467.

Heck, K.; Van Belle, G. \& Simberloff, D. 1975. Explicit calculation of the rarefaction diversity measurement and the determination of sufficient sample size. Ecology 56(6):1459-1461.

Hammer, Ø., Harper, D. \& RyAn, P. 2001. PAST: Paleontological statistics software package for education and data analysis. Palaeontologia Electronica 4(1):1-9.

Hoffmann, A. \& Blows, M. 1994. Species borders: ecological and evolutionary perspectives. Trends in Ecology and Evolution 9(6):223227.

Iriarte, A. 2007. Mamíferos de Chile. Barcelona, Lynx Ediciones. 420p.

KitzBERGER, T. 2012. Ecotones as complex arenas of disturbance, climate and human impacts: the trans-Andean forest-steppe ecotone of northern Patagonia. In: Myster, R. W. ed. Ecotones between forest and grassland. New York, Springer, p. 59-88.

Kohn, M. \& WaYne, R. 1997. Facts from feces revisited. Trends in Ecology and Evolution 12(6):223-227

LesiCA, P. \& Allendorf, F. 1995. When are peripheral populations valuable for conservation? Conservation Biology 9(4):753-760.

Luebert, F. \& Pliscoff, P. 2006. Sinopsis bioclimática y vegetacional de Chile. Santiago de Chile, Editorial Universitaria. 316p.

Mann, G. 1945. Mamíferos de Tarapacá. Observaciones realizadas durante una expedición al Alto Norte de Chile. Biológica 2:23-134.
MANN, G. 1978. Los pequeños mamíferos de Chile (marsupiales, quirópteros, edentados y roedores). Gayana Zoología 40:1-342.

Mella, J. 1999. Vertebrados terrestres posibles de encontrar en la XI región de Aysén. Aysén, Servicio Agrícola y Ganadero. 71p.

Moleón, M.; Sánchez-Zapata, J.; Sebastián-González, E. \& OwenSмIth, N. 2015. Carcass size shapes the structure and functioning of an African scavenging assemblage. Oikos 124:1391-1403.

PEÑA, L. 1988. Introducción a los Insectos de Chile. Santiago de Chile, Editorial Universitaria. 254p.

PoljaK, S.; Confalonieri, V.; Fasanella, M.; Gabrielli, M. \& Lizarralde, M. 2010. Phylogeography of the armadillo Chaetophractus villosus (Dasypodidae Xenarthra): post-glacial range expansion from Pampas to Patagonia (Argentina). Molecular Phylogenetics and Evolution 55(1):38-46.

Rojas, E. \& Gallardo, R. 2004. Manual de insectos asociados a la madera en la zona sur de Chile. Chile, Servicio Agrícola y Ganadero (SAG), Ministerio de Agricultura. 34p.

Selva, N.; JęDrzejeWska, B.; JęDrzeJeWski, W. \& WajRaK, A. 2005. Factors affecting carcass use by a guild of scavengers in European temperate Woodland. Canadian Journal of Zoology 83:1590-1601.

Soibelzon, E.; Daniele, G.; Negrete, J.; Carlini, A. \& Plischuk, S. 2007. Annual diet of the little hairy armadillo, Chaetophractus vellerosus (Mammalia, Dasypodidae), in Buenos Aires Province, Argentina. Journal of Mammalogy 88(5):1319-1324.

Superina, M. \& ABBA, A. 2014. Zaedyus pichiy (Cingulata: Dasypodidae). Mammalian Species 46(905):1-10.

Superina, M.; Fernández, F.; Stevani, E. \& Carrara, R. 2009. Summer diet of the pichi Zaedyus pichiy (Xenarthra: Dasypodidae) in Mendoza Province, Argentina. Journal of Arid Environments 73:683-686.

Talmage, R. \& Buchanan, G. 1954. The armadillo (Dasypus novemcinctus). A review of its natural history, ecology, anatomy and reproductive physiology. Rice Institute Pamphlet, Monographs in Biology 41(2):1-135.

TAMAYO, M. 2009. Descripciones de las especies vivientes (Orden Xenarthra). In: Muñoz-Pedreros, A. \& Yañez, J. eds. Mamíferos de Chile. Valdivia, CEA Ediciones, p. 85-91.

Tome, A.; TeIllier, S. \& Howorth, R. 2007. Contribución al conocimiento de la flora vascular de la Reserva Nacional Tamango, región de Aisén, Chile. Boletín del Museo Nacional de Historia Natural, Chile 56:9-25.

Toro, H.; ChiApa, E. \& ToBar, C. 2003. Biología de insectos. Valparaíso, Ediciones Universitarias de Valparaíso, Pontificia Universidad Católica de Valparaíso. 473p.

Travaini, T.; Donazar, J.; Rodriguez, A.; Ceballos, O.; Funes, M.; Delibes, M. \& Hiraldo, F. 1998. Use of european hare (Lepus europaeus) carcasses by an avian scavenging assemblage in Patagonia. Journal of Zoology 246(2):175-181.

Tyler, J.; Molinaro, S. \& Messina, M. 1996. A note on spring food of armadillos, Dasypus novemcinctus in a residential area. Proceedings of the Oklahoma Academy of Science 76:99-101.

Whitaker, J.; Ruckdeschel, C. \& BAKKen, L. 2012. Food of the armadillo Dasypus novemcinctus L. from Cumberland Island, GA. Southeastern Naturalist 11(3):487-506.

Winemiller, K. \& PiANKA, E. 1990. Organization in natural assemblages of desert lizards and tropical fishes. Ecological Monographs 60(1):27-55.

Wolffsohn, J. 1921. Catálogo de cráneos de mamíferos de Chile colectados entre los años 1896 y 1918. Revista Chilena de Historia Natural 25:511-529.

Yañez, J.; Cattan, P. \& Iriarte, A. 2009. Descripción de las especies vivientes: Mamíferos exóticos de Chile. In: Muñoz-Pedreros, A. \& Yañez, J. eds. Mamíferos de Chile. Valdivia, CEA Ediciones, p. 251-267.

ZIELINSKI, W. \& DUNCAN, N. 2004. Diets of sympatric populations of american martens (Martes americana) and fishers (Martes pennanti) in California. Journal of Mammalogy 85(3):470-477. 